MATHEMATICS OF COMPUTATION

Volume 70, Number 236, Pages 1383-1396

S 0025-5718(00)01289-8

Article electronically published on November 17, 2000

\title{
A POSTERIORI ERROR ESTIMATOR FOR A MIXED FINITE ELEMENT METHOD FOR REISSNER-MINDLIN PLATE
}

\author{
ELSA LIBERMAN
}

\begin{abstract}
We present an a posteriori error estimator for a mixed finite element method for the Reissner-Mindlin plate model. The finite element method we deal with, was analyzed by Durán and Liberman in 1992 and can also be seen as a particular example of the general family analyzed by Brezzi, Fortin, and Stenberg in 1991. The estimator is based on the evaluation of the residual of the finite element solution. We show that the estimator yields local lower and global upper bounds of the error in the numerical solution in a natural norm for the problem, which includes the $H^{1}$ norms of the terms corresponding to the deflection and the rotation and a dual norm for the shearing force. The estimates are valid uniformly with respect to the plate thickness.
\end{abstract}

\section{INTRODUCTION}

In the implementation of numerical methods for approximation of partial differential equations, the definition of a posteriori error estimators is the basic tool for adaptive mesh-refinement techniques, necessary when we are in the presence of local singularities of the solution.

In this paper we present an a posteriori error estimator for the finite element approximation of the Reissner-Mindlin plate model, which describes the displacement of a plate with moderate thickness subject to a transverse load. The definition of the estimator is based on the evaluation of the residual of the finite element solution.

Several a posteriori error estimators have been defined for different linear and nonlinear elliptic problems by using the residual equations (see for example [3, 4, $5,14,19,20$ )

For a fixed plate thickness the Reissner Mindlin plate model is a linear elliptic problem. But for small thickness the ellipticity constant deteriorates and makes the treatment of the problem difficult. In particular, in the definition of an estimator the main difficulty is the attainment of equivalence with an error norm independently of the plate thickness. To the author's knowledge, an estimator with this property has not yet been defined.

For the numerical solution of the Reissner-Mindlin equations, there are several mixed finite element methods which present good approximations of the solutions [2, 6, 7, 9, 10, 11, 15, 17] and are free from locking [8, 11, 12, 15, 17.

Received by the editor April 27, 1999 and, in revised form, January 6, 2000.

2000 Mathematics Subject Classification. Primary 65N30, 65N15, 74K20.

Member of C.I.C, Provincia de Buenos Aires, Argentina. 
We present an a posteriori error estimator for a method analyzed in 15 which can also be seen as a particular example of the general family analyzed in [12].

We define the error estimator for the $H^{1}$ norm of the deflection and the rotation, and for a sum of norms for the shear force, which includes the $\mathbf{H}_{0}(r o t)^{\prime}$ norm, and show that it yields local lower and global upper bounds of the error in the numerical solution, valid uniformly with respect to the plate thickness. It must be remarked that even though these norms are natural for the problem (in particular the inf - sup condition holds for the $\mathbf{H}_{0}(r o t)^{\prime}$ norm [11] and, when $t \rightarrow 0, \mathbf{H}_{0}(r o t)^{\prime}$ becomes the appropriate space for the shear), convergence for the shear force in this dual norm has not been proved, as far as we know. The results hold for any polygonal domain and, therefore, our estimator can be used for adaptive refinement when corner singularities arise.

The rest of the paper is organized as follows. In Section 2 we introduce the Reissner-Mindlin model and we analyze its approximation with the finite element method. We also give an additional a priori estimate related with the $L^{2}$ norm of the error in the rotor of the shear force. For the sake of clarity we divide the definition and analysis of the estimator in two sections. In Section 3 we define a weak norm for the error in the rotation and in the shear force and obtain estimates for this norm. Finally in Section 4, we define the estimator for the whole error and show the corresponding relations between the estimator and the natural error norm using the results of the previous section.

\section{The Reissner-Mindlin EQUations AND MIXED FINITE ELEMENT APPROXIMATION}

We use boldface type to denote vector quantities.

Let $\Omega \times[-t / 2, t / 2]$ be the region occupied by the undeformed elastic plate of thickness $0<t<1$, where $\Omega \subset R^{2}$ is a simply connected polygon.

Let us denote by $w$ and $\boldsymbol{\beta}$ the transverse displacement of the midsection of the plate and the rotation of fibers normal to it, respectively. Then, assuming for simplicity that the plate is clamped along the boundary of $\Omega$, the Reissner-Mindlin problem is:

Find $w \in H_{0}^{1}(\Omega)$ and $\boldsymbol{\beta} \in \mathbf{H}_{0}^{1}(\Omega)$ such that

$$
t^{3} a(\boldsymbol{\beta}, \boldsymbol{\eta})+\lambda t(\nabla w-\boldsymbol{\beta}, \nabla \zeta-\boldsymbol{\eta})=(g, \zeta) \quad \forall \boldsymbol{\eta} \in \mathbf{H}_{0}^{1}(\Omega), \forall \zeta \in H_{0}^{1}(\Omega),
$$

where ( , ) denotes the scalar product in either $L^{2}(\Omega)$ or $\mathbf{L}^{2}(\Omega)$, and $a(\boldsymbol{\beta}, \boldsymbol{\eta})$ is a coercive and continuous bilinear form defined by

$$
a(\boldsymbol{\beta}, \boldsymbol{\eta})=\frac{E}{12\left(1-\nu^{2}\right)} \int_{\Omega} D \Xi(\boldsymbol{\beta}): \Xi(\boldsymbol{\eta}),
$$

where $\Xi(\boldsymbol{\eta})$ is the symmetric part of the gradient of $\boldsymbol{\eta}, D$ is defined by

$$
D \Upsilon=[(1-\nu) \Upsilon+\nu \operatorname{tr}(\Upsilon) I],
$$

$E$ is the Young modulus, $\nu$ is the Poisson ratio, $\lambda=E k / 2(1+\nu)$, where $k$ is the shear correction factor, and $g$ represents the transverse load.

To analyze the problem for small values of $t, g$ is scaled in the form $g=t^{3} f$ so that the solution tends to a nonzero limit as $t$ tends to zero [11]. Taking, for the sake of simplicity, $\lambda=1$ and introducing

$$
\gamma=t^{-2}(\nabla w-\boldsymbol{\beta})
$$


equation (2.1) can be written equivalently as

$$
\begin{cases}a(\boldsymbol{\beta}, \boldsymbol{\eta})+(\boldsymbol{\gamma}, \nabla \zeta-\boldsymbol{\eta})=(f, \zeta) \quad \forall \boldsymbol{\eta} \in \mathbf{H}_{0}^{1}(\Omega), \forall \zeta \in H_{0}^{1}(\Omega), \\ t^{2}(\boldsymbol{\gamma}, \boldsymbol{\chi})-(\nabla w-\boldsymbol{\beta}, \boldsymbol{\chi})=0 \quad \forall \boldsymbol{\chi} \in \mathbf{L}^{2}(\Omega),\end{cases}
$$

which in the limit $t \rightarrow 0$ takes the form of a saddle point problem.

Let

$$
\mathbf{H}_{0}(\operatorname{rot}, \Omega)=\left\{\boldsymbol{\chi} \in \mathbf{L}^{2}(\Omega): \operatorname{rot}(\boldsymbol{\chi}) \in L^{2}(\Omega) \text { and } \boldsymbol{\chi} \cdot \boldsymbol{\tau}=0 \text { on } \partial \Omega\right\},
$$

where $\partial \Omega$ denotes the boundary of $\Omega$ and $\boldsymbol{\tau}$ is the unit tangent to the boundary, with the norm

$$
\|\chi\|_{\mathbf{H}_{0}(\text { rot }, \Omega)}:=\|\chi\|_{0}+\|\operatorname{rot} \chi\|_{0} .
$$

The following proposition, which is proved in 11], gives a decomposition for any $\chi \in \mathbf{H}_{0}($ rot,$\Omega)$, showing also that $\gamma \in \mathbf{H}_{0}($ rot,$\Omega)$.

Proposition 2.1. Let $B$ be defined on $\mathbf{H}_{0}^{1}(\Omega) \times H_{0}^{1}(\Omega)$ by

$$
B:(\boldsymbol{\eta}, \zeta) \longrightarrow(\nabla \zeta-\boldsymbol{\eta})
$$

The mapping $B$ is surjective onto the space $\mathbf{H}_{0}(r o t, \Omega)$, and for every $\boldsymbol{\chi} \in \mathbf{H}_{0}(\operatorname{rot}, \boldsymbol{\Omega})$ there exists $(\boldsymbol{\eta}, \zeta) \in \mathbf{H}_{0}^{1}(\Omega) \times H_{0}^{1}(\Omega)$ such that

$$
\chi=\nabla \zeta-\boldsymbol{\eta}
$$

and

$$
\|\nabla \zeta\|_{0}+\|\boldsymbol{\eta}\|_{1} \leq C\left\{\|\chi\|_{0}+\|\operatorname{rot} \boldsymbol{\chi}\|_{0}\right\}
$$

with $C$ independent of $\chi$.

As we stated in the Introduction, we will also consider the space

$$
\boldsymbol{\Gamma}=\mathbf{H}_{0}(\operatorname{rot}, \boldsymbol{\Omega})^{\prime}=\left\{\chi \in \mathbf{H}^{-1}(\boldsymbol{\Omega}) / \operatorname{div} \chi \in H^{-1}(\boldsymbol{\Omega})\right\}
$$

with the norm

$$
\|\chi\|_{\Gamma}^{2}=\|\chi\|_{-1}^{2}+\|\operatorname{div} \chi\|_{-1}^{2},
$$

which is equivalent to the dual norm.

Remark 2.1. From this and Proposition 2.1, it immediately follows that the following inf - sup condition holds:

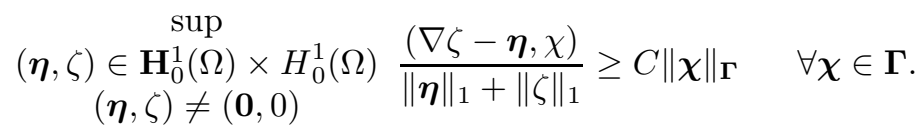

We describe now the finite element method for the Reissner-Mindlin model that we will consider. 
Let $\left\{\mathcal{T}_{h}\right\}_{0<h<1}$ be a regular family of triangulations of $\Omega$, where $h$ stands for the maximum diameter of the elements in the triangulation $\mathcal{T}_{h}$. In order to define the mixed finite element approximation, we have to give finite element spaces for the rotations, the transverse displacement, and the shear strain. Also we have to define an operator, usually some kind of interpolation, in order to relax the discrete equation corresponding to (2.2).

We use the standard notation $\mathcal{P}_{m}$ for the space of polynomials of degree less than or equal to $m$ and set $\mathbf{P}_{m}=\mathcal{P}_{m} \times \mathcal{P}_{m}$.

Given an element $T$, let $\left\{\lambda_{i}\right\}_{1 \leq i \leq 3}$ be its barycentric coordinates and $\boldsymbol{\tau}_{i}$ be the tangential vector to the edge $\partial T_{i}$ where $\lambda_{i}=0$. We define,

$$
\phi_{1}=\lambda_{2} \lambda_{3} \tau_{1}, \quad \phi_{2}=\lambda_{3} \lambda_{1} \tau_{2} \quad \text { and } \quad \phi_{3}=\lambda_{1} \lambda_{2} \tau_{3}
$$

then the finite element spaces for the method, $\mathbf{H}_{h} \subset \mathbf{H}_{0}^{1}(\Omega)$ for the rotations, $W_{h} \subset H_{0}^{1}(\Omega)$ for the transverse displacement, and $\boldsymbol{\Gamma}_{h} \subset \mathbf{L}^{2}(\Omega)$ for the shear strain, are defined as follows:

$$
\begin{gathered}
\mathbf{H}_{h}=\left\{\boldsymbol{\eta}_{h} \in \mathbf{H}_{0}^{1}(\Omega):\left.\boldsymbol{\eta}_{h}\right|_{T} \in \mathbf{P}_{1} \oplus \operatorname{span}\left\{\boldsymbol{\phi}_{1}, \boldsymbol{\phi}_{2}, \boldsymbol{\phi}_{3}\right\}, \forall T \in \mathcal{T}_{h}\right\}, \\
W_{h}=\left\{\zeta_{h} \in H_{0}^{1}(\Omega):\left.\zeta_{h}\right|_{T} \in \mathcal{P}_{1}, \forall T \in \mathcal{T}_{h}\right\}
\end{gathered}
$$

and $\boldsymbol{\Gamma}_{h}$ is a rotation of the lowest order Raviart-Thomas space [1],

$$
\boldsymbol{\Gamma}_{h}=\left\{\boldsymbol{\eta}_{h} \in \mathbf{H}_{0}(\operatorname{rot}, \Omega):\left.\boldsymbol{\eta}_{h}\right|_{T} \in \mathbf{P}_{0} \oplus\left(x_{2},-x_{1}\right) \mathcal{P}_{0}, \forall T \in \mathcal{T}_{h}\right\} .
$$

In particular, the inclusion

$$
\nabla W_{h} \subset \boldsymbol{\Gamma}_{h}
$$

holds.

The interpolation operator for this method $\boldsymbol{\Pi}: \mathbf{H}_{0}(\operatorname{rot}, \Omega) \cap \mathbf{H}^{1} \longrightarrow \boldsymbol{\Gamma}_{h}$ is defined by $\left.\boldsymbol{\Pi} \boldsymbol{\eta}\right|_{T}=\boldsymbol{\eta}_{I}$, where $\boldsymbol{\eta}_{I}$ is such that

$$
\int_{\partial T_{i}} \boldsymbol{\eta}_{I} \cdot \boldsymbol{\tau}_{i}=\int_{\partial T_{i}} \boldsymbol{\eta} \cdot \boldsymbol{\tau}_{i}, \quad i=1,2,3
$$

and which satisfies (see 11] for example)

$$
\|\boldsymbol{\eta}-\Pi \boldsymbol{\eta}\|_{0} \leq C h\|\boldsymbol{\eta}\|_{1} \quad \forall \boldsymbol{\eta} \in \mathbf{H}_{0}^{1}(\Omega) .
$$

Therefore the approximate solution $\left(\boldsymbol{\beta}_{h}, w_{h}, \boldsymbol{\gamma}_{h}\right) \in \mathbf{H}_{h} \times W_{h} \times \boldsymbol{\Gamma}_{h}$ is defined by

$$
\left\{\begin{array}{l}
a\left(\boldsymbol{\beta}_{h}, \boldsymbol{\eta}_{h}\right)+\left(\boldsymbol{\gamma}_{h}, \nabla \zeta_{h}-\boldsymbol{\Pi} \boldsymbol{\eta}_{h}\right)=\left(f, \zeta_{h}\right), \quad \forall \boldsymbol{\eta}_{h} \in \mathbf{H}_{h}, \quad \forall \zeta_{h} \in W_{h} \\
\boldsymbol{\gamma}_{h}=t^{-2}\left(\nabla w_{h}-\boldsymbol{\Pi} \boldsymbol{\beta}_{h}\right) .
\end{array}\right.
$$

Hereafter, $C$ denotes a constant which could depend on the minimum angle of the triangulation but is independent of the thickness $t$ and the meshsize $h$, and the symbol $\|$.$\| denotes a norm over the region \Omega$, if no explicit reference to the region is made. 
For the error in the approximation of the Reissner-Mindlin model with this method, the following a priori estimates are known [12], [15]:

$$
\left\|\boldsymbol{\beta}-\boldsymbol{\beta}_{h}\right\|_{1}+t\left\|\boldsymbol{\gamma}-\boldsymbol{\gamma}_{h}\right\|_{0}+\left\|w-w_{h}\right\|_{1} \leq C h\left\{\|\boldsymbol{\beta}\|_{2}+t\|\boldsymbol{\gamma}\|_{1}+\|\boldsymbol{\gamma}\|_{0}\right\}
$$

and also [12,

$$
\left\|\boldsymbol{\gamma}-\boldsymbol{\gamma}_{h}\right\|_{-1} \leq C h\left\{\|\boldsymbol{\beta}\|_{2}+t\|\gamma\|_{1}+\|\gamma\|_{0}\right\}
$$

and when $\Omega$ is a convex polygon [2],

$$
\|\boldsymbol{\beta}\|_{2}+t\|\boldsymbol{\gamma}\|_{1}+\|\boldsymbol{\gamma}\|_{0} \leq C\|f\|_{0} .
$$

Taking into account the definition of the norm in $\mathbf{H}_{0}(\operatorname{rot}, \Omega)$, we add to this a priori estimates the following one related with $\left\|\operatorname{rot}\left(\boldsymbol{\gamma}-\gamma_{h}\right)\right\|_{0}$.

Lemma 2.1. For the method defined previously it holds that

$$
t^{2}\left\|\operatorname{rot}\left(\boldsymbol{\gamma}-\gamma_{h}\right)\right\|_{0} \leq C h\left\{\|\boldsymbol{\beta}\|_{2}+t\|\boldsymbol{\gamma}\|_{1}+\|\boldsymbol{\gamma}\|_{0}\right\} .
$$

Proof. From the definition of $\gamma$ and $\gamma_{h}$ it follows that

$$
t^{2} \operatorname{rot}\left(\boldsymbol{\gamma}-\boldsymbol{\gamma}_{h}\right)=-\operatorname{rot}\left(\boldsymbol{\beta}-\boldsymbol{\Pi} \boldsymbol{\beta}_{h}\right) .
$$

Then

$$
t^{2}\left\|\operatorname{rot}\left(\boldsymbol{\gamma}-\boldsymbol{\gamma}_{h}\right)\right\|_{0} \leq\|\operatorname{rot}(\boldsymbol{\beta}-\boldsymbol{\Pi} \boldsymbol{\beta})\|_{0}+\left\|\operatorname{rot}\left(\boldsymbol{\Pi} \boldsymbol{\beta}-\boldsymbol{\Pi} \boldsymbol{\beta}_{h}\right)\right\|_{0} .
$$

It is known [1] that for $\boldsymbol{\eta} \in \mathbf{H}_{0}(\operatorname{rot}, \Omega)$,

$$
\operatorname{rot}(\boldsymbol{\Pi} \boldsymbol{\eta})=\operatorname{Prot}(\boldsymbol{\eta})
$$

where $P$ denotes the $L^{2}$ projection operator into $Q_{h}:=\operatorname{rot}\left(\boldsymbol{\Gamma}_{h}\right)$ and

$$
\|\operatorname{rot}(\boldsymbol{\eta}-\boldsymbol{\Pi} \boldsymbol{\eta})\|_{0} \leq C h\|\boldsymbol{\eta}\|_{2} .
$$

From (2.13), (2.14) and (2.15) we obtain

$$
t^{2}\left\|\operatorname{rot}\left(\boldsymbol{\gamma}-\gamma_{h}\right)\right\|_{0} \leq C\left\{h\|\boldsymbol{\beta}\|_{2}+\left\|\boldsymbol{\beta}-\boldsymbol{\beta}_{h}\right\|_{1}\right\},
$$

then (2.12) follows from the a priori estimates in (2.9).

\section{Preliminary error estimates}

According to the approximation results in the previous section and our remarks in the Introduction, our aim is to give an estimator for the following sum of errors:

$$
\left\|\nabla w-\nabla w_{h}\right\|_{0}+\left\|\boldsymbol{\beta}-\boldsymbol{\beta}_{h}\right\|_{1}+t^{2}\left\|\operatorname{rot}\left(\boldsymbol{\gamma}-\boldsymbol{\gamma}_{h}\right)\right\|_{0}+t\left\|\boldsymbol{\gamma}-\boldsymbol{\gamma}_{h}\right\|_{0}+\left\|\boldsymbol{\gamma}-\boldsymbol{\gamma}_{h}\right\|_{\boldsymbol{\Gamma}} .
$$

However, for the sake of clarity, we have divided the definition and analysis of the estimator in two sections. In this section we give, as a preliminary result, estimates for the errors in the rotation and the shear force in a weak norm. 
We define the weak norm for the error in $\boldsymbol{\beta}$ and $\boldsymbol{\gamma}$, as the dual norm in $\mathbf{H}_{0}^{1}(\Omega) \times$ $H_{0}^{1}(\Omega)$ of the operator $a\left(\boldsymbol{\beta}-\boldsymbol{\beta}_{h}, \boldsymbol{\eta}\right)+\left(\boldsymbol{\gamma}-\boldsymbol{\gamma}_{h}, \nabla \zeta-\boldsymbol{\eta}\right)$; that is,

$$
\begin{aligned}
& \left\|\left(\boldsymbol{\beta}-\boldsymbol{\beta}_{h}\right),\left(\boldsymbol{\gamma}-\boldsymbol{\gamma}_{h}\right)\right\|_{*, \boldsymbol{\Omega}} \\
& \quad \sup ^{(\boldsymbol{\eta}, \zeta) \in \mathbf{H}_{0}^{1}(\Omega) \times H_{0}^{1}(\Omega)} \frac{\left|a\left(\boldsymbol{\beta}-\boldsymbol{\beta}_{h}, \boldsymbol{\eta}\right)+\left(\boldsymbol{\gamma}-\boldsymbol{\gamma}_{h}, \nabla \zeta-\boldsymbol{\eta}\right)\right|}{\|\boldsymbol{\eta}\|_{1}+\|\zeta\|_{1}} . \\
& \quad \begin{array}{l}
\boldsymbol{\eta}, \zeta) \neq(\mathbf{0}, 0) \\
\quad
\end{array}
\end{aligned}
$$

Let $k$ be a fixed integer, $k \geq 1$. The estimator for this error norm is defined for any $T \in \mathcal{T}_{h}$ as

$$
\begin{aligned}
\varepsilon_{T}=\{ & \left\|P^{k} f\right\|_{0, T}^{2}|T|+\frac{1}{2} \sum_{\partial T_{i} \subset \partial T}\left\|\left[\boldsymbol{\gamma}_{h} \mathbf{n}_{i}\right]_{J}\right\|_{0, \partial T_{i}}^{2}\left|\partial T_{i}\right| \\
& \left.+\left\|\operatorname{div} D \Xi\left(\boldsymbol{\beta}_{h}\right)+\gamma_{h}\right\|_{0, T}^{2}|T|+\frac{1}{2} \sum_{\partial T_{i} \subset \partial T}\left\|\left[D \Xi\left(\boldsymbol{\beta}_{h}\right) \mathbf{n}_{i}\right]_{J}\right\|_{0, \partial T_{i}}^{2}\left|\partial T_{i}\right|\right\}^{1 / 2},
\end{aligned}
$$

where $P^{k}$ is the $L^{2}$ projection onto $\mathcal{P}_{k},|T|$ and $\left|\partial T_{i}\right|$ are the area of $T$ and the length of $\partial T_{i}, \mathbf{n}_{i}$ is the normal vector to the edge $\partial T_{i}$, and [.] $]_{J}$ denote the jump of the corresponding function across $\partial T_{i}$.

For each $T \in \mathcal{T}_{h}$ let

$$
\omega_{T}=\left\{\bigcup \tilde{T} \in \mathcal{T}_{h}: T \cap \tilde{T} \neq \emptyset\right\}
$$

The following theorem shows the equivalence between the error norm and the estimator.

Theorem 3.1. There exist two constants $C_{1}$ and $C_{2}$, depending on the minimum angle of the mesh, such that

$$
\left\|\left(\boldsymbol{\beta}-\boldsymbol{\beta}_{h}\right),\left(\boldsymbol{\gamma}-\gamma_{h}\right)\right\|_{*} \leq C_{1}\left\{\sum_{T \in \mathcal{T}_{h}}\left[\varepsilon_{T}^{2}+\left\|f-P^{k} f\right\|_{0, T}^{2}|T|\right]\right\}^{1 / 2}
$$

and

$$
\varepsilon_{T} \leq C_{2}\left\{\left\|\left(\boldsymbol{\beta}-\boldsymbol{\beta}_{h}\right),\left(\boldsymbol{\gamma}-\boldsymbol{\gamma}_{h}\right)\right\|_{*, \omega_{T}}+\sum_{\tilde{T} \in \omega_{T}}|\tilde{T}|^{1 / 2}\left\|f-P^{k} f\right\|_{0, \tilde{T}}\right\}
$$

Proof. From (2.3) we have

$$
a\left(\boldsymbol{\beta}-\boldsymbol{\beta}_{h}, \boldsymbol{\eta}\right)+\left(\boldsymbol{\gamma}-\boldsymbol{\gamma}_{h}, \nabla \zeta-\boldsymbol{\eta}\right)=(f, \zeta)-a\left(\boldsymbol{\beta}_{h}, \boldsymbol{\eta}\right)-\left(\boldsymbol{\gamma}_{h}, \nabla \zeta-\boldsymbol{\eta}\right) .
$$

For $\psi \in H_{0}^{1}(\Omega)$ or $\mathbf{H}_{0}^{1}(\Omega)$ we denote by $\psi_{I} \in H_{0}^{1}(\Omega)$ or $\mathbf{H}_{0}^{1}(\Omega)$, respectively, a piecewise linear average interpolant, as defined in [13, 18], satisfying

$$
\left\|\psi-\psi_{I}\right\|_{0} \leq C h\|\psi\|_{1}
$$

and

$$
\left\|\psi_{I}\right\|_{1} \leq C\|\psi\|_{1}
$$


Taking $\boldsymbol{\eta}_{h}=\boldsymbol{\eta}_{I}$ and $\zeta_{h}=\zeta_{I}$ in (2.8) and subtracting it from (3.5) we get $(3.8)$

$$
\begin{aligned}
& a\left(\boldsymbol{\beta}-\boldsymbol{\beta}_{h}, \boldsymbol{\eta}\right)+\left(\boldsymbol{\gamma}-\boldsymbol{\gamma}_{h}, \nabla \zeta-\boldsymbol{\eta}\right) \\
& =\left(f, \zeta-\zeta_{I}\right)-a\left(\boldsymbol{\beta}_{h}, \boldsymbol{\eta}-\boldsymbol{\eta}_{I}\right)-\left(\boldsymbol{\gamma}_{h},(\nabla \zeta-\boldsymbol{\eta})-\left(\nabla \zeta_{I}-\boldsymbol{\eta}_{I}\right)\right)+\left(\boldsymbol{\gamma}_{h}, \boldsymbol{\eta}_{I}-\boldsymbol{\Pi} \boldsymbol{\eta}_{I}\right) \\
& =\sum_{T \in \mathcal{T}_{h}}\left\{\left(f, \zeta-\zeta_{I}\right)_{T}-\frac{1}{2} \sum_{\partial T_{i} \subset \partial T} \int_{\partial T_{i}}\left[\gamma_{h} \mathbf{n}_{i}\right]_{J}\left(\zeta-\zeta_{I}\right)\right. \\
& +\left(\operatorname{div} D \Xi\left(\boldsymbol{\beta}_{h}\right)+\boldsymbol{\gamma}_{h}, \boldsymbol{\eta}-\boldsymbol{\eta}_{I}\right)_{T} \\
& \left.-\frac{1}{2} \sum_{\partial T_{i} \subset \partial T} \int_{\partial T_{i}}\left[D \Xi\left(\boldsymbol{\beta}_{h}\right) \mathbf{n}_{i}\right]_{J}\left(\boldsymbol{\eta}-\boldsymbol{\eta}_{I}\right)\right\} \\
& +\left(\gamma_{h}, \boldsymbol{\eta}_{I}-\boldsymbol{\Pi} \boldsymbol{\eta}_{I}\right) \\
& \leq \sum_{T \in \mathcal{T}_{h}}\left\{\|f\|_{0, T}\left\|\zeta-\zeta_{I}\right\|_{0, T}+\frac{1}{2} \sum_{\partial T_{i} \subset \partial T}\left\|\left[\gamma_{h} \mathbf{n}_{i}\right]_{J}\right\|_{0, \partial T_{i}}\left\|\zeta-\zeta_{I}\right\|_{0, \partial T_{i}}\right. \\
& +\left\|\operatorname{div} D \Xi\left(\boldsymbol{\beta}_{h}\right)+\gamma_{h}\right\|_{0, T}\left\|\boldsymbol{\eta}-\boldsymbol{\eta}_{I}\right\|_{0, T} \\
& \left.+\frac{1}{2} \sum_{\partial T_{i} \subset \partial T}\left\|\left[D \Xi\left(\boldsymbol{\beta}_{h}\right) \mathbf{n}_{i}\right]_{J}\right\|_{0, \partial T_{i}}\left\|\boldsymbol{\eta}-\boldsymbol{\eta}_{I}\right\|_{0, \partial T_{i}}\right\} \\
& +\left(\gamma_{h}, \boldsymbol{\eta}_{I}-\Pi \boldsymbol{\eta}_{I}\right) \\
& \leq C\left\{\sum_{T \in \mathcal{T}_{h}}\left[\varepsilon_{T}^{2}+|T|\left\|f-P^{k} f\right\|_{0, T}^{2}\right]\right\}^{1 / 2}\left\{\|\boldsymbol{\eta}\|_{1}+\|\zeta\|_{1}\right\}+\left(\boldsymbol{\gamma}_{h}, \boldsymbol{\eta}_{I}-\boldsymbol{\Pi} \boldsymbol{\eta}_{I}\right) .
\end{aligned}
$$

We are going now to bound the term $\left(\boldsymbol{\gamma}_{h}, \boldsymbol{\eta}_{I}-\boldsymbol{\Pi} \boldsymbol{\eta}_{I}\right)$.

It is known ([16], Lemma 3.3) that for $\boldsymbol{\eta}_{I}$ as defined above, there exists $\phi \in H_{0}^{1}(\Omega)$ such that $\left.\phi\right|_{T} \in \mathcal{P}_{2}$ and

$$
\nabla \phi=\boldsymbol{\eta}_{I}-\Pi \boldsymbol{\eta}_{I}
$$

In [16] it is also proved that $\phi$ vanishes at all the nodes of the triangulation. Hence, an usual scaling argument on each element $T$ yields

$$
\|\phi\|_{0, \partial T_{i}} \leq C\left|\partial T_{i}\right|^{1 / 2}\|\nabla \phi\|_{0, T}
$$

Then

$$
\begin{aligned}
\left(\gamma_{h}, \boldsymbol{\eta}_{I}-\boldsymbol{\Pi} \boldsymbol{\eta}_{I}\right) & =\left(\gamma_{h}, \nabla \phi\right)=\sum_{T \in \mathcal{T}_{h}} \frac{1}{2} \sum_{\partial T_{i} \subset \partial T} \int_{\partial T_{i}}\left[\gamma_{h} \mathbf{n}_{i}\right]_{J} \phi \\
& \leq \sum_{T \in \mathcal{T}_{h}} \frac{1}{2} \sum_{\partial T_{i} \subset \partial T}\left\|\left[\gamma_{h} \mathbf{n}_{i}\right]_{J}\right\|_{0, \partial T_{i}}\|\phi\|_{0, \partial T_{i}} \\
& \leq C \sum_{T \in \mathcal{T}_{h}}\left\{\frac{1}{2} \sum_{\partial T_{i} \subset \partial T}\left\|\left[\gamma_{h} \mathbf{n}_{i}\right]_{J}\right\|_{0, \partial T_{i}}\left|\partial T_{i}\right|^{1 / 2}\right\}\|\nabla \phi\|_{0, T} \\
& \leq C\left\{\sum_{T \in \mathcal{T}_{h}}\left[\frac{1}{2} \sum_{\partial T_{i} \subset \partial T}\left\|\left[\gamma_{h} \mathbf{n}_{i}\right]_{J}\right\|_{0, \partial T_{i}}^{2}\left|\partial T_{i} \| T\right|\right]\right\}^{1 / 2}\|\boldsymbol{\eta}\|_{1}
\end{aligned}
$$

where we have used (2.7) and (3.7) to obtain the last inequality. 
This shows that the last inner product in (3.8) can be bounded by the previous terms of the same expression.

From (3.8) and (3.9) we obtain

$$
\frac{\left|a\left(\boldsymbol{\beta}-\boldsymbol{\beta}_{h}, \boldsymbol{\eta}\right)+\left(\boldsymbol{\gamma}-\boldsymbol{\gamma}_{h}, \nabla \zeta-\boldsymbol{\eta}\right)\right|}{\|\boldsymbol{\eta}\|_{1}+\|\zeta\|_{1}} \leq C\left\{\sum_{T \in \mathcal{T}_{h}}\left[\varepsilon_{T}^{2}+\left\|f-P^{k} f\right\|_{0, T}^{2}|T|\right]\right\}^{1 / 2}
$$

from which it follows 3.3 .

In order to prove inequality (3.4) we need the following lemma.

Lemma 3.1. Let $T \in \mathcal{T}_{h}$. Given $\mathbf{q} \in \mathbf{L}^{2}(T), \mathbf{p} \in \mathbf{L}^{2}(\partial T)$, there exists $\hat{\boldsymbol{\eta}}_{T} \in \mathbf{P}_{k+3}$ such that

$$
\left\{\begin{array}{l}
\left(\hat{\boldsymbol{\eta}}_{T}, \mathbf{r}\right)_{T}=(\mathbf{q}, \mathbf{r})_{T} \quad \forall \mathbf{r} \in \mathbf{P}_{k}(T) \\
\int_{\partial T_{i}} \hat{\boldsymbol{\eta}}_{T} \mathbf{s}=\int_{\partial T_{i}} \mathbf{p s} \quad \forall \mathbf{s} \in \mathbf{P}_{k+1}(\partial T) \\
\hat{\boldsymbol{\eta}}_{T}=\mathbf{0} \quad \text { at the vertices of } T,
\end{array}\right.
$$

and

$$
\left\|\hat{\boldsymbol{\eta}}_{T}\right\|_{0, T} \leq C\left\{\|\mathbf{q}\|_{0, T}+\sum_{\partial T_{i} \subset \partial T}\left|\partial T_{i}\right|^{1 / 2}\|\mathbf{p}\|_{0, \partial T_{i}}\right\} .
$$

In particular if $\mathbf{p}=\mathbf{0}$, then $\left.\hat{\boldsymbol{\eta}}_{T}\right|_{\partial T}=\mathbf{0}$.

Proof. The proof follows with arguments similar to those given in [1].

In particular, the previous result is also valid for scalar functions:

Lemma 3.2. Let $T \in \mathcal{T}_{h}$. Given $q \in L^{2}(T), p \in L^{2}(\partial T)$, there exists $\hat{\zeta}_{T} \in \mathcal{P}_{k+3}$ such that

$$
\left\{\begin{array}{l}
\left(\hat{\zeta}_{T}, r\right)_{T}=(q, r)_{T} \quad \forall r \in \mathcal{P}_{k}(T) \\
\int_{\partial T_{i}} \hat{\zeta}_{T} s=\int_{\partial T_{i}} p s \quad \forall s \in \mathcal{P}_{k+1}(\partial T) \\
\hat{\zeta}_{T}=0 \quad \text { at the vertices of } T
\end{array}\right.
$$

and

$$
\left\|\hat{\zeta}_{T}\right\|_{0, T} \leq C\left\{\|q\|_{0, T}+\sum_{\partial T_{i} \subset \partial T}\left|\partial T_{i}\right|^{1 / 2}\|p\|_{0, \partial T_{i}}\right\}
$$

In particular if $p=0$, then $\left.\hat{\zeta}_{T}\right|_{\partial T}=0$.

Now for fixed $T \in \mathcal{T}_{h}$, we take

$$
\begin{aligned}
& q=P^{k} f|T| \in \mathcal{P}_{k}(T), \\
& \left.p\right|_{\partial T_{i}}=\frac{1}{4}\left|\partial T_{i}\right|\left[\gamma_{h} \mathbf{n}_{i}\right]_{J}, \quad p \in \mathcal{P}_{1}(\partial T),
\end{aligned}
$$


and we take the corresponding $\hat{\zeta}_{T}$ defined in Lemma 3.2, making appropriate modifications when $T$ intercepts $\partial \Omega$; whereas for each $\tilde{T} \in \omega_{T}, \tilde{T} \neq T,\left.\hat{\zeta}\right|_{\tilde{T}}$ is defined by the same lemma taking now

$$
\begin{aligned}
& q=0, \\
& \left.p\right|_{\partial T_{i}}=\left\{\begin{array}{l}
0 \text { if } \partial \tilde{T}_{i} \cap \partial T=\emptyset, \\
\text { the same as in (3.15) if } \partial \tilde{T}_{i} \cap \partial T \neq \emptyset .
\end{array}\right.
\end{aligned}
$$

Let $\hat{\zeta}$ be defined such that $\left.\hat{\zeta}\right|_{T}=\hat{\zeta}_{T}$ if $T \in \omega_{T}$ and 0 is outside of $\omega_{T}$. From its definition we see that $\hat{\zeta} \in H_{0}^{1}(\Omega)$, and

$$
\begin{aligned}
& \left\|P^{k} f\right\|_{0, T}^{2}|T|+\frac{1}{4} \sum_{\partial T_{i} \subset \partial T}\left\|\left[\gamma_{h} \mathbf{n}_{i}\right]_{J}\right\|_{0, \partial T_{i}}^{2} \|\left|\partial T_{i}\right| \\
& \quad=\sum_{\tilde{T} \in \omega_{T}}\left\{\left(\gamma_{h}, \nabla \hat{\zeta}\right)_{\tilde{T}}+\left(P^{k} f, \hat{\zeta}\right)_{\tilde{T}}\right\}=\left(\gamma_{h}-\gamma, \nabla \hat{\zeta}\right)_{\omega_{T}}+\sum_{\tilde{T} \in \omega_{T}}\left(P^{k} f-f, \hat{\zeta}\right)_{\tilde{T}} .
\end{aligned}
$$

For the same fixed $T$ we proceed in the same way and determine $\hat{\boldsymbol{\eta}}_{T}$ applying (3.11) for

$$
\begin{aligned}
& \mathbf{q}=-\left(\operatorname{div} D \Xi\left(\boldsymbol{\beta}_{h}\right)+\gamma_{h}\right)|T| \quad \in \mathbf{P}_{1}(T), \\
& \left.\mathbf{p}\right|_{\partial T_{i}}=\frac{1}{4}\left[D \Xi\left(\boldsymbol{\beta}_{h}\right) \mathbf{n}_{i}\right]_{J}\left|\partial T_{i}\right|, \quad \mathbf{p} \in \mathbf{P}_{1}(\partial T),
\end{aligned}
$$

and $\hat{\boldsymbol{\eta}}_{\tilde{T}}$ for $\tilde{T} \in \omega_{T}, \tilde{T} \neq T$, making the corresponding modifications as in (3.16).

Let $\hat{\boldsymbol{\eta}} \in \mathbf{H}_{0}^{1}(\Omega)$ be defined as $\left.\hat{\boldsymbol{\eta}}\right|_{T}=\hat{\boldsymbol{\eta}}_{T}$ if $T \in \omega_{T}$ and $\mathbf{0}$ are outside of $\omega_{T}$. Then

$$
\begin{aligned}
& \left\|\operatorname{div} D \Xi\left(\boldsymbol{\beta}_{h}\right)+\gamma_{h}\right\|_{0, T}^{2}|T|+\frac{1}{4} \sum_{\partial T_{i} \subset \partial T}\left\|\left[D \Xi\left(\boldsymbol{\beta}_{h}\right) \mathbf{n}_{i}\right]_{J}\right\|_{0, \partial T_{i}}^{2}\left|\partial T_{i}\right| \\
& =\sum_{\tilde{T} \in \omega_{T}}\left\{a\left(\boldsymbol{\beta}_{h}, \hat{\boldsymbol{\eta}}\right)_{\tilde{T}}-\left(\boldsymbol{\gamma}_{h}, \hat{\boldsymbol{\eta}}\right)_{\tilde{T}}\right\}=a\left(\boldsymbol{\beta}_{h}-\boldsymbol{\beta}, \hat{\boldsymbol{\eta}}\right)_{\omega_{T}}+\left(\boldsymbol{\gamma}_{h}-\boldsymbol{\gamma},-\hat{\boldsymbol{\eta}}\right)_{\omega_{T}} .
\end{aligned}
$$

Adding (3.17) and (3.19) we obtain

$$
\begin{aligned}
& \frac{\varepsilon_{T}^{2}}{\|\hat{\zeta}\|_{1, \omega_{T}}+\|\hat{\boldsymbol{\eta}}\|_{1, \omega_{T}}} \\
& \leq C\left\{\frac{\left|a\left(\boldsymbol{\beta}-\boldsymbol{\beta}_{h}, \hat{\boldsymbol{\eta}}\right)_{\omega_{T}}+\left(\boldsymbol{\gamma}-\gamma_{h}, \nabla \hat{\zeta}-\hat{\boldsymbol{\eta}}\right)_{\omega_{T}}\right|}{\|\hat{\zeta}\|_{1, \omega_{T}}+\|\hat{\boldsymbol{\eta}}\|_{1, \omega_{T}}}+\frac{\sum_{\tilde{T} \epsilon_{\omega_{T}}}\left(P^{k} f-f, \hat{\zeta}\right)_{\tilde{T}}}{\|\hat{\zeta}\|_{1, \omega_{T}}+\|\hat{\boldsymbol{\eta}}\|_{1, \omega_{T}}}\right\} \\
& \leq C\left\{\left\|\left(\boldsymbol{\beta}-\boldsymbol{\beta}_{h}\right),\left(\boldsymbol{\gamma}-\boldsymbol{\gamma}_{h}\right)\right\|_{\omega_{,} \omega_{T}}+\frac{\sum_{\tilde{T} \in \omega_{T}}\left\|P^{k} f-f\right\|_{0, \tilde{T}}\|\hat{\zeta}\|_{0, \tilde{T}}}{\|\hat{\zeta}\|_{1, \omega_{T}}+\|\hat{\boldsymbol{\eta}}\|_{1, \omega_{T}}}\right\} .
\end{aligned}
$$

Replacing (3.15) in (3.14) and (3.18) in (3.12), we get the bound

$$
\|\hat{\zeta}\|_{0, \tilde{T}}+\|\hat{\boldsymbol{\eta}}\|_{0, \tilde{T}} \leq C|\tilde{T}|^{1 / 2} \varepsilon_{T} \text { for } \tilde{T} \in \omega_{T},
$$

and by standard scaling arguments we also get

$$
\|\hat{\zeta}\|_{1, \omega_{T}}+\|\hat{\boldsymbol{\eta}}\|_{1, \omega_{T}} \leq C \varepsilon_{T} .
$$


Using these bounds in (3.20), it follows that

$$
\varepsilon_{T} \leq C\left\{\left\|\left(\boldsymbol{\beta}-\boldsymbol{\beta}_{h}\right),\left(\boldsymbol{\gamma}-\gamma_{h}\right)\right\|_{*, \omega_{T}}+\sum_{\tilde{T} \in \omega_{T}}|\tilde{T}|^{1 / 2}\left\|P^{k} f-f\right\|_{0, \tilde{T}}\right\},
$$

so the theorem is proved.

\section{Error estimator}

Now we are able to define an estimator for the whole error. For any $T \in \mathcal{T}_{h}$ we define it as

$$
\eta_{T}=\left\{\varepsilon_{T}^{2}+\left\|\boldsymbol{\beta}_{h}-\boldsymbol{\Pi} \boldsymbol{\beta}_{h}\right\|_{0, T}^{2}+\left\|\operatorname{rot}\left(\boldsymbol{\beta}_{h}-\boldsymbol{\Pi} \boldsymbol{\beta}_{h}\right)\right\|_{0, T}^{2}\right\}^{1 / 2} .
$$

Before coming to the main result of equivalence we need the following proposition.

Proposition 4.1. There exists a constant $C$ such that

$$
\begin{aligned}
& \left\|\nabla w-\nabla w_{h}\right\|_{0}+\left\|\boldsymbol{\beta}-\boldsymbol{\beta}_{h}\right\|_{1}+t\left\|\boldsymbol{\gamma}-\gamma_{h}\right\|_{0}+t^{2}\left\|\operatorname{rot}\left(\boldsymbol{\gamma}-\boldsymbol{\gamma}_{h}\right)\right\|_{0} \\
& \leq C\left\{\sum_{T \in \mathcal{T}_{h}}\left[\eta_{T}^{2}+\left\|f-P^{k} f\right\|_{0, T}^{2}|T|\right]\right\}^{1 / 2}
\end{aligned}
$$

Proof. Consider the expression

$$
\frac{\left|a\left(\boldsymbol{\beta}-\boldsymbol{\beta}_{h}, \boldsymbol{\eta}\right)+\left(\boldsymbol{\gamma}-\boldsymbol{\gamma}_{h}, \nabla \zeta-\boldsymbol{\eta}\right)\right|}{\|\boldsymbol{\eta}\|_{1}+\|\zeta\|_{1}} .
$$

If we replace in (4.3) $\zeta=w-w_{h}$ and $\boldsymbol{\eta}=\boldsymbol{\beta}-\boldsymbol{\beta}_{h}$, we obtain

$$
\begin{aligned}
& \frac{\left|a\left(\boldsymbol{\beta}-\boldsymbol{\beta}_{h}, \boldsymbol{\beta}-\boldsymbol{\beta}_{h}\right)+t^{2}\left\|\boldsymbol{\gamma}-\boldsymbol{\gamma}_{h}\right\|_{0}^{2}+\left(\boldsymbol{\gamma}-\boldsymbol{\gamma}_{h}, \boldsymbol{\beta}_{h}-\boldsymbol{\Pi} \boldsymbol{\beta}_{h}\right)\right|}{\left\|\boldsymbol{\beta}-\boldsymbol{\beta}_{h}\right\|_{1}+\left\|\nabla w-\nabla w_{h}\right\|_{0}} \\
& \leq\left\|\left(\boldsymbol{\beta}-\boldsymbol{\beta}_{h}\right),\left(\boldsymbol{\gamma}-\gamma_{h}\right)\right\|_{*} .
\end{aligned}
$$

Taking into account that $\boldsymbol{\beta}_{h}-\boldsymbol{\Pi} \boldsymbol{\beta}_{h} \in \mathbf{H}_{0}(r o t, \Omega)$, and according to Proposition 2.1, there exist $\phi \in \mathbf{H}_{0}^{1}(\Omega)$ and $\psi \in H_{0}^{1}(\Omega)$ such that

$$
\boldsymbol{\beta}_{h}-\boldsymbol{\Pi} \boldsymbol{\beta}_{h}=\nabla \psi-\boldsymbol{\phi},
$$

with

$$
\|\nabla \psi\|_{0}+\|\phi\|_{1} \leq C\left\{\left\|\boldsymbol{\beta}_{h}-\boldsymbol{\Pi} \boldsymbol{\beta}_{h}\right\|_{0}+\left\|\operatorname{rot}\left(\boldsymbol{\beta}_{h}-\boldsymbol{\Pi} \boldsymbol{\beta}_{h}\right)\right\|_{0}\right\} .
$$

Replacing again in (4.3) $\zeta=\psi$ and $\boldsymbol{\eta}=\boldsymbol{\phi}$, and using (4.6) we get

$$
\begin{aligned}
& \frac{\left|a\left(\boldsymbol{\beta}-\boldsymbol{\beta}_{h}, \boldsymbol{\phi}\right)+\left(\boldsymbol{\gamma}-\boldsymbol{\gamma}_{h}, \boldsymbol{\beta}_{h}-\boldsymbol{\Pi} \boldsymbol{\beta}_{h}\right)\right|}{\left\|\boldsymbol{\beta}_{h}-\boldsymbol{\Pi} \boldsymbol{\beta}_{h}\right\|_{0}+\left\|\operatorname{rot}\left(\boldsymbol{\beta}_{h}-\boldsymbol{\Pi} \boldsymbol{\beta}_{h}\right)\right\|_{0}} \leq C \frac{\left|a\left(\boldsymbol{\beta}-\boldsymbol{\beta}_{h}, \boldsymbol{\phi}\right)+\left(\gamma-\boldsymbol{\gamma}_{h}, \nabla \psi-\boldsymbol{\phi}\right)\right|}{\|\nabla \psi\|_{0}+\|\boldsymbol{\phi}\|_{1}} \\
& \quad \leq C\left\|\left(\boldsymbol{\beta}-\boldsymbol{\beta}_{h}\right),\left(\boldsymbol{\gamma}-\boldsymbol{\gamma}_{h}\right)\right\|_{*} .
\end{aligned}
$$


Then

$(4.8)$

$$
\begin{aligned}
& \left|\left(\gamma-\gamma_{h}, \boldsymbol{\beta}_{h}-\boldsymbol{\Pi} \boldsymbol{\beta}_{h}\right)\right| \\
& \leq C\left\{\left\|\left(\boldsymbol{\beta}-\boldsymbol{\beta}_{h}\right),\left(\boldsymbol{\gamma}-\boldsymbol{\gamma}_{h}\right)\right\|_{*}\left[\left\|\boldsymbol{\beta}_{h}-\boldsymbol{\Pi} \boldsymbol{\beta}_{h}\right\|_{0}+\left\|\operatorname{rot}\left(\boldsymbol{\beta}_{h}-\boldsymbol{\Pi} \boldsymbol{\beta}_{h}\right)\right\|_{0}\right]+\left\|\boldsymbol{\beta}-\boldsymbol{\beta}_{h}\right\|_{1}\|\boldsymbol{\phi}\|_{1}\right\} \\
& \leq C\left\{\left\|\left(\boldsymbol{\beta}-\boldsymbol{\beta}_{h}\right),\left(\boldsymbol{\gamma}-\boldsymbol{\gamma}_{h}\right)\right\|_{*}\left[\left\|\boldsymbol{\beta}_{h}-\boldsymbol{\Pi} \boldsymbol{\beta}_{h}\right\|_{0}+\left\|\operatorname{rot}\left(\boldsymbol{\beta}_{h}-\boldsymbol{\Pi} \boldsymbol{\beta}_{h}\right)\right\|_{0}\right]\right. \\
& \left.+\left[\left\|\boldsymbol{\beta}_{h}-\boldsymbol{\Pi} \boldsymbol{\beta}_{h}\right\|_{0}+\left\|\operatorname{rot}\left(\boldsymbol{\beta}_{h}-\boldsymbol{\Pi} \boldsymbol{\beta}_{h}\right)\right\|_{0}\right]\left\|\boldsymbol{\beta}-\boldsymbol{\beta}_{h}\right\|_{1}\right\} \\
& \leq C\left\{\left[\left\|\left(\boldsymbol{\beta}-\boldsymbol{\beta}_{h}\right),\left(\gamma-\gamma_{h}\right)\right\|_{*}+\left\|\boldsymbol{\beta}_{h}-\boldsymbol{\Pi} \boldsymbol{\beta}_{h}\right\|_{0}+\left\|\operatorname{rot}\left(\boldsymbol{\beta}_{h}-\boldsymbol{\Pi} \boldsymbol{\beta}_{h}\right)\right\|_{0}\right]\right. \\
& \left.\times\left[\left\|\boldsymbol{\beta}-\boldsymbol{\beta}_{h}\right\|_{1}+\left\|\nabla w-\nabla w_{h}\right\|_{0}+\left\|\boldsymbol{\beta}_{h}-\boldsymbol{\Pi} \boldsymbol{\beta}_{h}\right\|_{0}+\left\|\operatorname{rot}\left(\boldsymbol{\beta}_{h}-\boldsymbol{\Pi} \boldsymbol{\beta}_{h}\right)\right\|_{0}\right]\right\},
\end{aligned}
$$

where we have used continuity of $a($,$) to obtain the first inequality, and (4.6) to$ bound $\|\phi\|_{1}$ in the second inequality.

Returning to (4.4) we can see that

$$
\begin{aligned}
& a\left(\boldsymbol{\beta}-\boldsymbol{\beta}_{h}, \boldsymbol{\beta}-\boldsymbol{\beta}_{h}\right)+t^{2}\left\|\boldsymbol{\gamma}-\boldsymbol{\gamma}_{h}\right\|_{0}^{2} \\
& \leq\left\|\left(\boldsymbol{\beta}-\boldsymbol{\beta}_{h}\right),\left(\boldsymbol{\gamma}-\boldsymbol{\gamma}_{h}\right)\right\|_{*}\left\{\left\|\boldsymbol{\beta}-\boldsymbol{\beta}_{h}\right\|_{1}+\left\|\nabla w-\nabla w_{h}\right\|_{0}\right. \\
& \left.\quad+\left\|\boldsymbol{\beta}_{h}-\boldsymbol{\Pi} \boldsymbol{\beta}_{h}\right\|_{0}+\left\|\operatorname{rot}\left(\boldsymbol{\beta}_{h}-\boldsymbol{\Pi} \boldsymbol{\beta}_{h}\right)\right\|_{0}\right\} \\
& \quad+\left|\left(\boldsymbol{\gamma}-\boldsymbol{\gamma}_{h}, \boldsymbol{\beta}_{h}-\boldsymbol{\Pi} \boldsymbol{\beta}_{h}\right)\right|,
\end{aligned}
$$

so, using the coerciveness of $a($,$) and (4.8) we also have$

$$
\begin{aligned}
& \left\|\boldsymbol{\beta}-\boldsymbol{\beta}_{h}\right\|_{1}^{2}+t^{2}\left\|\boldsymbol{\gamma}-\boldsymbol{\gamma}_{h}\right\|_{0}^{2} \\
& \leq C\left\{\left\|\left(\boldsymbol{\beta}-\boldsymbol{\beta}_{h}\right),\left(\gamma-\gamma_{h}\right)\right\|_{*}+\left\|\boldsymbol{\beta}_{h}-\boldsymbol{\Pi} \boldsymbol{\beta}_{h}\right\|_{0}+\| \operatorname{rot}\left(\boldsymbol{\beta}_{h}-\boldsymbol{\Pi} \boldsymbol{\beta}_{h} \|_{0}\right\}\right. \\
& \quad \times\left\{\left\|\boldsymbol{\beta}-\boldsymbol{\beta}_{h}\right\|_{1}+\left\|\nabla w-\nabla w_{h}\right\|_{0}+\left\|\boldsymbol{\beta}_{h}-\boldsymbol{\Pi} \boldsymbol{\beta}_{h}\right\|_{0}+\left\|\operatorname{rot}\left(\boldsymbol{\beta}_{h}-\Pi \boldsymbol{\beta}_{h}\right)\right\|_{0}\right\} .
\end{aligned}
$$

From the definition of $\gamma$ and $\gamma_{h}$ we have the following identity:

$$
\nabla w-\nabla w_{h}=t^{2}\left(\boldsymbol{\gamma}-\boldsymbol{\gamma}_{h}\right)+\left(\boldsymbol{\beta}-\boldsymbol{\beta}_{h}\right)+\left(\boldsymbol{\beta}_{h}-\boldsymbol{\Pi} \boldsymbol{\beta}_{h}\right),
$$

from which it follows that

$$
\left\|\nabla w-\nabla w_{h}\right\|_{0} \leq t\left\|\boldsymbol{\gamma}-\boldsymbol{\gamma}_{h}\right\|_{0}+\left\|\boldsymbol{\beta}-\boldsymbol{\beta}_{h}\right\|_{1}+\left\|\boldsymbol{\beta}_{h}-\boldsymbol{\Pi} \boldsymbol{\beta}_{h}\right\|_{0} .
$$

Adding $\left\|\boldsymbol{\beta}_{h}-\boldsymbol{\Pi} \boldsymbol{\beta}_{h}\right\|_{0}^{2}+\left\|\operatorname{rot}\left(\boldsymbol{\beta}_{h}-\boldsymbol{\Pi} \boldsymbol{\beta}_{h}\right)\right\|_{0}^{2}$ to both members in (4.10) and making use of (4.12) we arrive at

$$
\begin{aligned}
& \left\|\boldsymbol{\beta}_{h}-\boldsymbol{\Pi} \boldsymbol{\beta}_{h}\right\|_{0}^{2}+\left\|\operatorname{rot}\left(\boldsymbol{\beta}_{h}-\boldsymbol{\Pi} \boldsymbol{\beta}_{h}\right)\right\|_{0}^{2}+\left\|\boldsymbol{\beta}-\boldsymbol{\beta}_{h}\right\|_{1}^{2}+t^{2}\left\|\boldsymbol{\gamma}-\gamma_{h}\right\|_{0}^{2} \\
& \leq C\left\{\left\|\left(\boldsymbol{\beta}-\boldsymbol{\beta}_{h}\right),\left(\gamma-\gamma_{h}\right)\right\|_{*}+\left\|\boldsymbol{\beta}_{h}-\boldsymbol{\Pi} \boldsymbol{\beta}_{h}\right\|_{0}+\left\|\operatorname{rot}\left(\boldsymbol{\beta}_{h}-\boldsymbol{\Pi} \boldsymbol{\beta}_{h}\right)\right\|_{0}\right\} \\
& \quad \times\left\{\left\|\boldsymbol{\beta}_{h}-\boldsymbol{\Pi} \boldsymbol{\beta}_{h}\right\|_{0}+\left\|\operatorname{rot}\left(\boldsymbol{\beta}_{h}-\boldsymbol{\Pi} \boldsymbol{\beta}_{h}\right)\right\|_{0}+\left\|\boldsymbol{\beta}-\boldsymbol{\beta}_{h}\right\|_{1}+t\left\|\boldsymbol{\gamma}-\boldsymbol{\gamma}_{h}\right\|_{0}\right\},
\end{aligned}
$$

from which we obtain

$$
\begin{aligned}
& \left\|\boldsymbol{\beta}_{h}-\boldsymbol{\Pi} \boldsymbol{\beta}_{h}\right\|_{0}+\left\|\operatorname{rot}\left(\boldsymbol{\beta}_{h}-\boldsymbol{\Pi} \boldsymbol{\beta}_{h}\right)\right\|_{0}+\left\|\boldsymbol{\beta}-\boldsymbol{\beta}_{h}\right\|_{1}+t\left\|\boldsymbol{\gamma}-\boldsymbol{\gamma}_{h}\right\|_{0} \\
& \leq C\left\{\left\|\left(\boldsymbol{\beta}-\boldsymbol{\beta}_{h}\right),\left(\gamma-\gamma_{h}\right)\right\|_{*}+\left\|\boldsymbol{\beta}_{h}-\boldsymbol{\Pi} \boldsymbol{\beta}_{h}\right\|_{0}+\left\|\operatorname{rot}\left(\boldsymbol{\beta}_{h}-\boldsymbol{\Pi} \boldsymbol{\beta}_{h}\right)\right\|_{0}\right\} .
\end{aligned}
$$


Also from (4.11) we have

$$
t^{2} \operatorname{rot}\left(\boldsymbol{\gamma}-\boldsymbol{\gamma}_{h}\right)=-\operatorname{rot}\left(\boldsymbol{\beta}-\boldsymbol{\beta}_{h}\right)-\operatorname{rot}\left(\boldsymbol{\beta}_{h}-\boldsymbol{\Pi} \boldsymbol{\beta}_{h}\right),
$$

from which

$$
t^{2}\left\|\operatorname{rot}\left(\boldsymbol{\gamma}-\boldsymbol{\gamma}_{h}\right)\right\|_{0} \leq\left\|\boldsymbol{\beta}-\boldsymbol{\beta}_{h}\right\|_{1}+\left\|\operatorname{rot}\left(\boldsymbol{\beta}_{h}-\boldsymbol{\Pi} \boldsymbol{\beta}_{h}\right)\right\|_{0} .
$$

From (4.12) and (4.16) we see that

$$
\begin{aligned}
& t^{2}\left\|\operatorname{rot}\left(\boldsymbol{\gamma}-\boldsymbol{\gamma}_{h}\right)\right\|_{0}+\left\|\nabla w-\nabla w_{h}\right\|_{0}+\left\|\boldsymbol{\beta}-\boldsymbol{\beta}_{h}\right\|_{1}+t\left\|\boldsymbol{\gamma}-\boldsymbol{\gamma}_{h}\right\|_{0} \\
& \leq C\left\{\left\|\boldsymbol{\beta}_{h}-\boldsymbol{\Pi} \boldsymbol{\beta}_{h}\right\|_{0}+\left\|\operatorname{rot}\left(\boldsymbol{\beta}_{h}-\mathbf{\Pi} \boldsymbol{\beta}_{h}\right)\right\|_{0}+\left\|\boldsymbol{\beta}-\boldsymbol{\beta}_{h}\right\|_{1}+t\left\|\boldsymbol{\gamma}-\boldsymbol{\gamma}_{h}\right\|_{0}\right\} .
\end{aligned}
$$

From this inequality and (4.14) we get

$$
\begin{aligned}
& t^{2}\left\|\operatorname{rot}\left(\boldsymbol{\gamma}-\boldsymbol{\gamma}_{h}\right)\right\|_{0}+\left\|\nabla w-\nabla w_{h}\right\|_{0}+\left\|\boldsymbol{\beta}-\boldsymbol{\beta}_{h}\right\|_{1}+t\left\|\boldsymbol{\gamma}-\boldsymbol{\gamma}_{h}\right\|_{0} \\
& \leq C\left\{\left\|\left(\boldsymbol{\beta}-\boldsymbol{\beta}_{h}\right),\left(\boldsymbol{\gamma}-\boldsymbol{\gamma}_{h}\right)\right\|_{*}+\left\|\boldsymbol{\beta}_{h}-\boldsymbol{\Pi} \boldsymbol{\beta}_{h}\right\|_{0}+\left\|\operatorname{rot}\left(\boldsymbol{\beta}_{h}-\boldsymbol{\Pi} \boldsymbol{\beta}_{h}\right)\right\|_{0}\right\} .
\end{aligned}
$$

Finally (4.2) follows easily from (4.18), using (3.3) and the definition of $\eta_{T}$.

In the following theorem we obtain the main result of equivalence between the estimator and the sum of errors.

Theorem 4.1. There exist two constants $C_{1}$ and $C_{2}$ depending on the minimum angle of the mesh such that

$$
\begin{aligned}
& \left\|\nabla w-\nabla w_{h}\right\|_{0}+\left\|\boldsymbol{\beta}-\boldsymbol{\beta}_{h}\right\|_{1}+t^{2}\left\|\operatorname{rot}\left(\boldsymbol{\gamma}-\gamma_{h}\right)\right\|_{0}+t\left\|\boldsymbol{\gamma}-\gamma_{h}\right\|_{0}+\left\|\boldsymbol{\gamma}-\gamma_{h}\right\|_{\boldsymbol{\Gamma}} \\
& \leq C_{1}\left\{\sum_{T \in \mathcal{T}_{h}}\left[\eta_{T}^{2}+\left\|f-P^{k} f\right\|_{0, T}^{2}|T|\right]\right\}^{1 / 2}
\end{aligned}
$$

and

$$
\begin{aligned}
\eta_{T} \leq C_{2}\left\{\left\|\left(\boldsymbol{\beta}-\boldsymbol{\beta}_{h}\right)\right\|_{1, \omega_{T}}+\left\|\nabla w-\nabla w_{h}\right\|_{0, \omega_{T}}+t^{2}\left\|\operatorname{rot}\left(\boldsymbol{\gamma}-\gamma_{h}\right)\right\|_{0, \omega_{T}}\right. \\
\left.+t\left\|\boldsymbol{\gamma}-\gamma_{h}\right\|_{0, \omega_{T}}+t\left\|\boldsymbol{\gamma}-\gamma_{h}\right\|_{\boldsymbol{\Gamma}, \omega_{T}}+\sum_{\tilde{T} \in \omega_{T}}|\tilde{T}|^{1 / 2}\left\|f-P^{k} f\right\|_{0, \tilde{T}}\right\} .
\end{aligned}
$$

Proof. For the proof of the first inequality, taking into account Proposition 4.1, we have to bound only $\left\|\gamma-\gamma_{h}\right\|_{\Gamma}$. For fixed $\boldsymbol{\eta}$

$$
\frac{a\left(\boldsymbol{\beta}-\boldsymbol{\beta}_{h}, \boldsymbol{\eta}\right)}{\|\boldsymbol{\eta}\|_{1}}+\frac{\left(\boldsymbol{\gamma}-\boldsymbol{\gamma}_{h},-\boldsymbol{\eta}\right)}{\|\boldsymbol{\eta}\|_{1}} \leq\left\|\left(\boldsymbol{\beta}-\boldsymbol{\beta}_{h}\right),\left(\boldsymbol{\gamma}-\boldsymbol{\gamma}_{h}\right)\right\|_{*} .
$$

From this,

$$
\left\|\boldsymbol{\gamma}-\gamma_{h}\right\|_{-1} \leq C\left\{\left\|\boldsymbol{\beta}-\boldsymbol{\beta}_{h}\right\|_{1}+\left\|\left(\boldsymbol{\beta}-\boldsymbol{\beta}_{h}\right),\left(\boldsymbol{\gamma}-\boldsymbol{\gamma}_{h}\right)\right\|_{*}\right\} .
$$

Also

$$
\left\|\operatorname{div}\left(\boldsymbol{\gamma}-\boldsymbol{\gamma}_{h}\right)\right\|_{-1} \leq\left\|\left(\boldsymbol{\beta}-\boldsymbol{\beta}_{h}\right),\left(\boldsymbol{\gamma}-\boldsymbol{\gamma}_{h}\right)\right\|_{*} .
$$

Combining (4.21), (4.22), and the results in Proposition 4.1 and Theorem 3.1 we arrive at (4.19). 
To obtain (4.20), we consider the bound for $\varepsilon_{T}$ from Theorem 3.1 and the following inequality:

$\left\|\left(\boldsymbol{\beta}-\boldsymbol{\beta}_{h}\right),\left(\boldsymbol{\gamma}-\boldsymbol{\gamma}_{h}\right)\right\|_{*, \omega_{T}} \leq\left\|\boldsymbol{\beta}-\boldsymbol{\beta}_{h}\right\|_{1, \omega_{T}}+\left\|\boldsymbol{\gamma}-\boldsymbol{\gamma}_{h}\right\|_{-1, \omega_{T}}+\left\|\operatorname{div}\left(\boldsymbol{\gamma}-\boldsymbol{\gamma}_{h}\right)\right\|_{-1, \omega_{T}}$.

On the other hand we obtain from (4.11)

$$
\left\|\boldsymbol{\beta}_{h}-\boldsymbol{\Pi} \boldsymbol{\beta}_{h}\right\|_{0, T} \leq\left\|\nabla w-\nabla w_{h}\right\|_{0, T}+\left\|\boldsymbol{\beta}-\boldsymbol{\beta}_{h}\right\|_{1, T}+t\left\|\boldsymbol{\gamma}-\gamma_{h}\right\|_{0, T},
$$

and from 4.15)

$$
\left\|\operatorname{rot}\left(\boldsymbol{\beta}_{h}-\boldsymbol{\Pi} \boldsymbol{\beta}_{h}\right)\right\|_{0, T} \leq t^{2}\left\|\operatorname{rot}\left(\boldsymbol{\gamma}-\boldsymbol{\gamma}_{h}\right)\right\|_{0, T}+\left\|\boldsymbol{\beta}-\boldsymbol{\beta}_{h}\right\|_{1, T} .
$$

The proof is completed by adding (3.4), (4.24) and (4.25) and making use of (4.23).

\section{ACKNOWLEDGMENT}

I want to thank Dr. M. Ainsworth, Dr. R. Durán and Dr. R. Rodriguez for their helpful comments.

\section{REFERENCES}

[1] D. N. ARnold And F. BrezzI, Mixed and non conforming finite element methods implementation, postprocessing and error estimates, R.A.I.R.O., Modél. Math. Anal. Numer. 19, 1985, pp. 7-32. MR 87g:65126

[2] D. N. ARnold and R. S. FAlK, A uniformly accurate finite element method for the ReissnerMindlin plate, SIAM J. Numer. Anal. 26 (1989), 1276-1290. MR 91c:65068

[3] I. BABUS̆KA AND A. Miller, A feedback finite element method with a posteriori error estimation. I. The finite element method and some basic properties of the a posteriori error estimator, Comp. Meth. Appl. Mech. Eng. 61 (1987), 1-40. MR 88d:73036

[4] I. Babus̆Ka And W. C. Rheinboldt, A posteriori error estimators in the finite element method, Int. J. Numer. Methods Eng. 12 (1978), 1587-1615.

[5] R. E. BANK AND A. Weiser, Some a posteriori error estimators for elliptic partial differential equations, Math. Comp. 44, (1985), 283-301. MR 86g:65207

[6] K. J. Bathe AND F. BREzzi, On the convergence of a four-node plate bending element based on Mindlin-Reissner plate theory and a mixed interpolation, MAFELAP V (J. R. Witheman, ed.), Academic Press, London, 1985, pp. 491-503. MR 87f:65125

[7] K. J. BAthe And F. BREzzI, A simplified analysis of two plate bending elements-the MITC4 and MITC9 elements, NUMETA 87 (G. N. Pande and J. Middleton, eds.), Numerical Techniques for Engineering Analysis and Design, vol. 1, Martinus Nijhoff, Dordrecht, 1987.

[8] K.J. Bathe, F. Brezzi and M. Fortin, Mixed-interpolated elements for Reissner-Mindlin plates, Internat. J. Numer. Methods Eng. 28 (1989), 1787-1801. MR 90g:73090

[9] K. J. Bathe And E. N. Dvorkin, A four-node plate bending element based on Mindlin Reissner plate theory and a mixed interpolation, J. Numer. Methods Engrg. 21 (1985),367383.

[10] F. Brezzi And M. Fortin, Numerical approximation of Mindlin-Reissner plates, Math. Comp. 47 (1986), 151-158. MR 87g:75057

[11] F. Brezzi And M. Fortin, Mixed and hybrid finite element methods, Springer-Verlag, New York (1991). MR 92d:65187

[12] F. Brezzi, M. Fortin And R. Stenberg, Error analysis of mixed-interpolated elements for Mindlin-Reissner plates, Math. Models Methods Appl. Sci. 1 (1991), 125-151. MR 92e:73030

[13] P. Clement, Approximation by finite element functions using local regularization. RAIRO Anal. Numér., 9 (1975) 77-84. MR 53:4569] 
[14] E. DARI, R. DuRÁN, C. PADRA, V. VAMPA A posteriori error estimators for nonconforming finite element methods, RAIRO Modél. Math. Anal. Numér. 30 (5) (1996), 385-400. MR 97f:65066

[15] R. Durán And E. Liberman, On mixed finite element methods for the Reissner-Mindlin plate model, Math. Comp. 58, Num. 198 (1992), 561-573. MR 92f:65135

[16] R. Durán, L. Hervella-Nieto, E. Liberman, R. Rodriguez and J. Solomin, Approximation of the vibration modes of a plate by Reissner-Mindlin equations, Math. Comp. 68 (1999), 1447-1463. MR 99m:73045

[17] P. Peisker AND D. BRAess, Uniform Convergence of Mixed Interpolated Elements for Reissner-Mindlin Plates, RAIRO Modél Math. Anal. Numér. 26 (5) (1992), 557-574. MR 93j: 73070

[18] L. R. Scott And S. Zhang, Finite element interpolation of nonsmooth functions satisfying boundary conditions, Math. Comp. 54 (1990) 483-493. MR 90j:65021

[19] R. Verfürth, A posteriori error estimators for the Stokes Equations, Numer. Math. 55 (1989), 309-325. MR 90d:65187

[20] R. Verfürth, A posteriori error estimates for nonlinear problems. Finite element discretizations of elliptic equations, Math. Comp. 62, Num. 206 (1994), 445-475. MR 94j:65136

Departamento de Matemática, Facultad de Ciencias Exactas, Universidad Nacional de La Plata, C.C.172, (1900) La Plata, Argentina

E-mail address: elsali@mate.unlp.edu.ar 